\title{
Que faire d'une bibliothèque allemande ? La longue gestation de la BNU après 1918
}

\section{Julien Gueslin}

\section{(2) OpenEdition}

1 Journals

Édition électronique

URL : http://journals.openedition.org/rbnu/1239

DOI : $10.4000 /$ rbnu. 1239

ISSN : 2679-6104

\section{Éditeur}

Bibliothèque nationale et universitaire de Strasbourg

\section{Édition imprimée}

Date de publication : 1 novembre 2016

Pagination : 96-105

ISBN : 9782859230630

ISSN : 2109-2761

\section{Référence électronique}

Julien Gueslin, «Que faire d'une bibliothèque allemande ? La longue gestation de la BNU après 1918 », La Revue de la BNU [En ligne], 14 | 2016, mis en ligne le 01 janvier 2020, consulté le 13 décembre 2020. URL : http://journals.openedition.org/rbnu/1239 ; DOI : https://doi.org/10.4000/rbnu.1239

\section{(c) (i) (2)}

La Revue de la BNU est mise à disposition selon les termes de la Licence Creative Commons Attribution - Pas d'Utilisation Commerciale - Partage dans les Mêmes Conditions 4.0 International. 


\section{Que faire d'une}

\section{bibliothèque allemande?}

\section{La longue gestation de la BNU après I9I8}

Le décret du 23 juillet 1926 a vu la naissance de la Bibliothèque nationale et universitaire de Strasbourg, qui reçoit alors un nouveau nom et un nouveau statut après huit longues années de gestation. Comme l'a bien montré L. Buchholzer dans sa synthèse récente, cette longue réflexion a permis de conférer à l'établissement des fondements durables ${ }^{1}$. S'appuyant sur les ordonnances de 1872 et de 1909, le nouvel établissement semble s'enraciner dans l'héritage allemand. Il conserve ainsi son indépendance juridique et son autonomie financière tout en s'adaptant au contexte français. Mais derrière la " façade " du nouveau statut, chaque article a fait l'objet d'âpres débats traduisant des divergences politiques, juridiques, bibliothéconomiques au sein de l'administration française, de l'opinion alsacienne et en premier lieu au sein de la bibliothèque.

L'utilisation de nouvelles sources non encore exploitées permet de mieux comprendre l'acuité des débats d'alors et la difficulté qu'on eut à trancher, qui fit retarder à tel point la solution qu'on craignit pour l'avenir de la bibliothèque, prise dans la tourmente financière du milieu des années vingt ${ }^{2}$. La question de la BNU, de son statut et du reclassement de son personnel fut considérée en 1926 par la Direction générale des services d'Alsace et de Lorraine comme " une des questions les plus importantes et les plus complexes " qu'elle ait eu à résoudre ${ }^{3}$. L'opinion alsacienne commença de plus à s'émouvoir face à un immobilisme qu'elle ne parvenait pas à comprendre. La question menaçait de prendre un tour de plus en plus politique et d'être utilisée dans le procès fait par une partie de l'opinion régionale contre ce qui semblait être du désintérêt, de l'impéritie voire une sourde hostilité qu'auraient nourrie les administrations parisiennes.
On évoquera donc ici en premier lieu la situation transitoire de la bibliothèque après 1918 , qui aboutit à une altération de sa situation antérieure, puis le débat sur son statut qui s'engage entre les différentes parties, et enfin la façon dont le statut du 23 juillet 1926 est le fruit d'un véritable compromis.

\section{Une assimilation en catimini ?}

On ne reviendra pas ici sur l'histoire des débuts de la présence française, fort bien évoquée par L. Buchholzer : en décembre 1919, l'administration française décide de renommer la bibliothèque "Bibliothèque universitaire et régionale " de Strasbourg (BURS), appellation qui persista dans les documents officiels et sur la façade du bâtiment jusqu'en 1926.

Mais selon la loi du 17 octobre 1919 sur le régime transitoire de l'Alsace-Lorraine, les dispositifs législatifs et réglementaires en vigueur avant 1918 continuent à régir la vie des territoires et des institutions se trouvant sur le sol alsacien jusqu'à l'introduction de la législation française par l'intermédiaire de lois spéciales. En théorie donc, la bibliothèque, si elle a changé de nom, conserve son ancien mode de fonctionnement et en premier lieu sa personnalité juridique et son autonomie financière, lui donnant son caractère d'établissement public indépendant, en particulier vis-à-vis de l'université. À plusieurs reprises, en 1919 et en 1923, des bibliothécaires déjà présents à l'époque allemande comme Charles Klein et Louis Wilhelm chercheront à attirer l'attention de l'administration sur ce fait, sans parvenir à leurs fins ${ }^{4}$. 


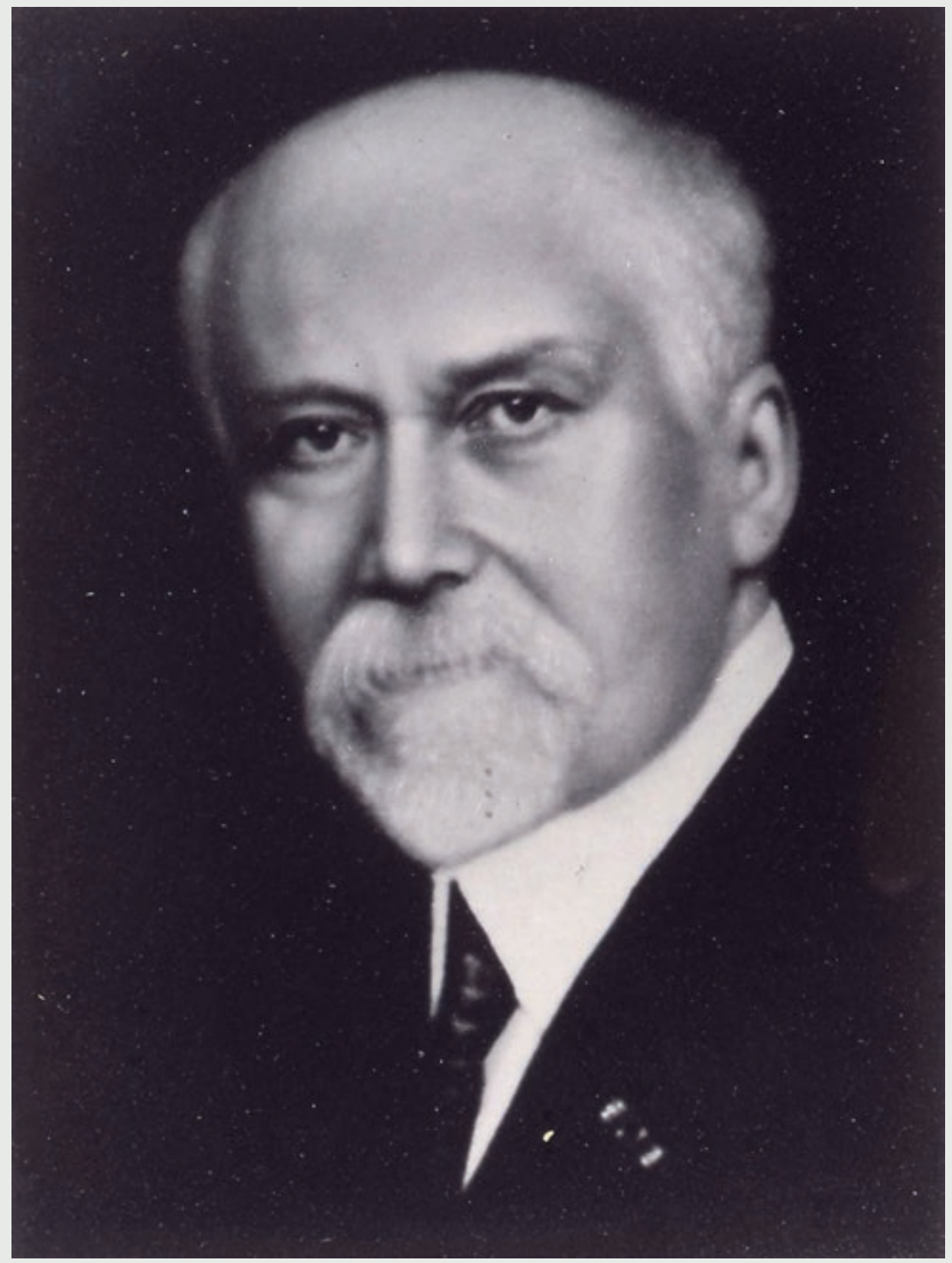

Raymond Poincaré (1860-1934), homme politique français, président de la République (1913-1920), président du Conseil à plusieurs reprises et président de la Société des Amis de l'Université de 1920 à 1934 , s'impliqua dans les débats alsaciens et en particulier dans celui sur la future BNU. Il fut le premier président de son conseil d'administration (coll. BNU). 


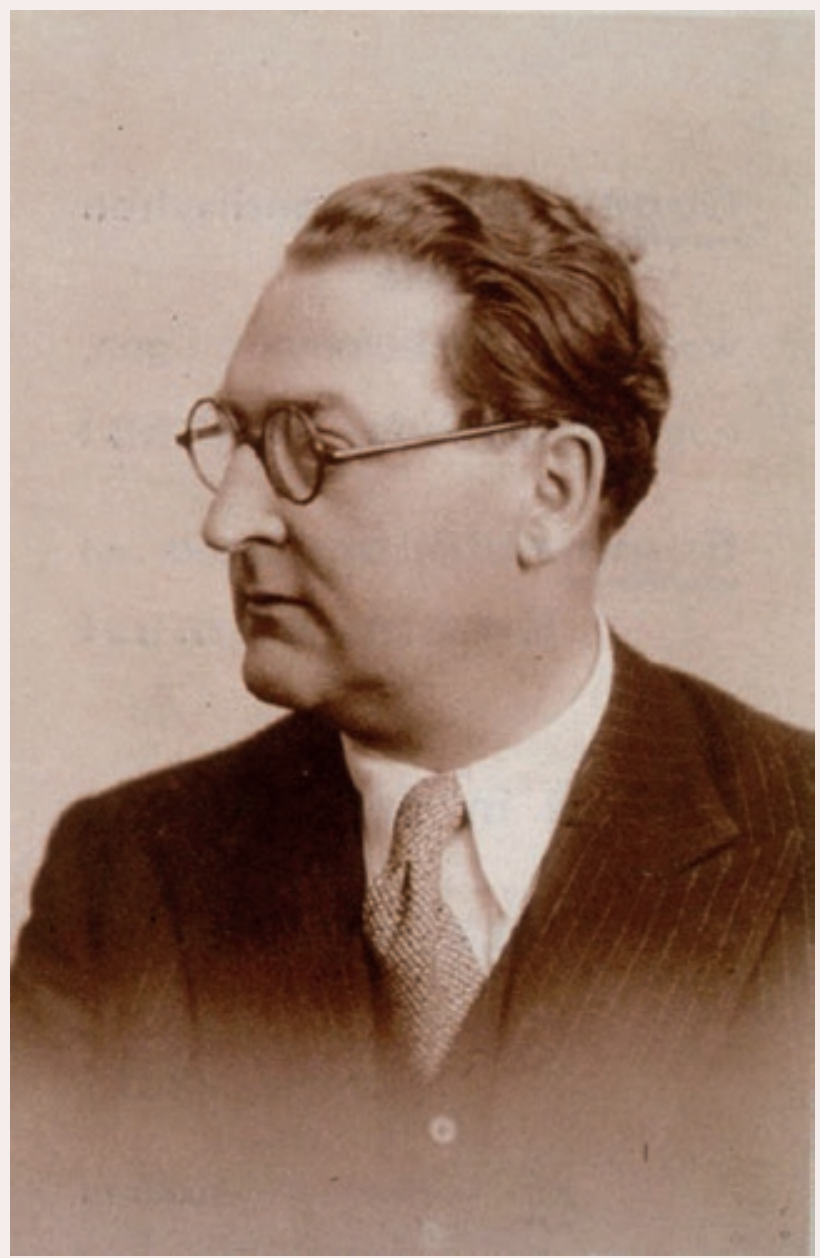

Charles Frey (1888-1955), député du Bas-Rhin et futur maire de Strasbourg, fut l'un des auteurs du projet de loi de 1924 visant à fixer le statut de la bibliothèque, puis l'un des interlocuteurs de l'administration pour la réflexion qui suivit (coll. BNU). 
Or très vite se produit une évolution opposée. La bibliothèque passe sous la tutelle de facto du recteur. Les crédits qu'elle reçoit sont versés par l'intermédiaire de l'université (sur un chapitre " Matériel de l'université "). Le budget de la bibliothèque n'est donc plus qu'une partie de celui des dépenses de l'université, la subvention d'État étant englobée dans les recettes de cette dernière et le Conseil de l'université arrêtant le montant des crédits attribués pour les acquisitions et le reste des dépenses. L'existence d'une commission de la bibliothèque composée d'universitaires (comme dans les autres universités françaises), mise en place pour discuter du règlement intérieur et de la politique d'acquisition, pose rapidement problème : est-elle simplement consultative ou a-t-elle un véritable rôle administratif ? Elle aboutit à des tensions croissantes entre des bibliothécaires se sentant remis en cause et un personnel universitaire ayant parfois l'impression d'être considéré comme un intrus dans la bibliothèque.

De plus, sans que cela soit dit explicitement, il y a aussi clairement une tension entre une partie du personnel alsacien s'érigeant en gardiens du temple et voyant dans l'établissement une expression du particularisme alsacien, et des universitaires et une partie de l'administration voulant préserver un tel " outil de travail " mais connaissant mal l'ancien statut, et qui le perçoivent à travers le prisme des traditions universitaires et bibliothéconomiques de l'époque. C'est la grande différence entre une université très majoritairement francisée et une bibliothèque qui a pu conserver une partie de son ancien personnel d'origine alsacienne.

Il est particulièrement intéressant de voir qu'il faudra attendre 1924 pour que les services centraux parisiens prennent en compte le fait qu'il n'y a pas besoin d'une loi pour établir la personnalité civile de la bibliothèque, tout simplement parce que celle-ci a été établie dans les ordonnances allemandes. Elle n'a donc jamais cessé d'exister, au moins sur le plan juridique, même si dans les faits elle est très " malmenée ".

Certains milieux alsaciens critiques voudront voir dans cette évolution illégale un " mépris absolu des textes $" 5$, voire un véritable passage en force d'administrations françaises voulant faire rentrer dans le rang une institution inclassable et lutter contre ce qui leur apparaît toujours à la fois comme la "Landesbibliothek " et un établissement national.

On ne trouve pas, dans la documentation dont on dispose, de trace d'une politique aussi déterminée, visant consciemment à « assimiler " progressivement la biblio- thèque et à en faire une bibliothèque universitaire classique. Il est certain que dès les premiers travaux pendant la guerre, l'idée a été de reconstituer une nouvelle université française dont le rayonnement ne pourrait être accru que par " l'instrument d'études " incomparable qu'était la KULB ${ }^{6}$. Mais depuis son poste de Commissaire général, Millerand invoquera lui-même le caractère " national " que la bibliothèque a vocation à avoir ${ }^{7}$. S'il ne rentre pas dans les détails du statut futur, cela symbolise quelque peu l'hésitation qui semble prédominer parmi l'administration française. Durant une période pleine d'incertitudes, c'est plus une politique provisoire, presque accidentelle, qui aboutit à cette évolution.

Avant 1919, c'est un personnel administratif supérieur, le "Curateur de l'université ", qui avait la charge du contrôle de la bibliothèque et de l'université ${ }^{8}$, tout en respectant strictement le caractère distinct des deux entités juridiques (le recteur, dans le système allemand, ne s'occupe que des affaires d'enseignement). Or comme il n'existe aucun équivalent de ce poste dans le système administratif français, c'est donc le recteur de l'université qui se retrouve progressivement investi de ces fonctions, aboutissant ainsi à une confusion des genres.

De même, utilisant ses prérogatives, le Commissariat général de la République se décide en 1920 à fixer par arrêté des cadres provisoires, afin de reclasser le personnel de droit local de la bibliothèque et de déterminer les traitements et conditions d'avancement. Le décret du 5 janvier 1923 aboutit à un nouveau reclassement, toujours provisoire. Dans la majorité des cas, on cherche au maximum à utiliser le système français, mais là encore, faute de correspondances, on se retrouve obligé de forger des catégories originales (ainsi celle de "bibliothécaires adjointes ") 9 .

Dans l'incertitude des premiers temps et compte tenu du rôle actif joué par le Commissariat général d'AlsaceLorraine, cette politique provisoire avait pu faire fi des tensions générées. La facilité des contacts, les crédits alloués par le Commissariat général, le souci de verser des subsides permettant de faire face quelque peu à l'inflation de l'après-guerre avaient pu donner l'illusion d'un effort maintenu auprès de la bibliothèque. Or avec la perte progressive du pouvoir du Commissariat général, qui va aboutir à sa disparition en 1925, l'inquiétude ne cesse de croître en Alsace du fait de l'éloignement des centres de décision à Paris et du manque apparent d'implication de ceux-ci. Dès 1922, le rattachement du budget d'AlsaceLorraine au budget général (les crédits à destination de la bibliothèque relevant désormais du ministère de 
l'Instruction publique) aboutit à une baisse considérable de celui de la bibliothèque et à un certain alignement avec les moyens limités d'alors des bibliothèques universitaires françaises de province. Si l'on ajoute la vive polémique ayant trait à l'abandon de l'ancien système de classement des livres pour un système considéré (à tort) comme français, on peut comprendre les craintes d'une partie importante des milieux politiques et intellectuels alsaciens, alertés par certains bibliothécaires, de voir très rapidement disparaître l'ancienne institution qui attirait auparavant des chercheurs du monde entier et faisait l'orgueil de la région.

Ä partir de 1924, la question va donc prendre un tour politique, avec l'implication croissante du monde politique alsacien qui s'empare du sujet et relaie l'inquiétude de l'opinion. Plusieurs bibliothécaires possèdent des liens étroits avec celui-ci, ainsi Louis Wilhelm ou Thérèse Kiener dont l'époux est un conseiller général fort influent. L'impatience gagne les parties intéressées, avec l'introduction de différents textes législatifs et réglementaires introduisant la législation française : loi du 22 juillet 1923 prévoyant l'incorporation des fonctionnaires alsaciens dans le cadre général, décrets du 30 mai 1924 déclarant applicables en Alsace de nombreuses dispositions législatives relatives au monde universitaire et constituant l'Université de Strasbourg. Or l'article 8 du dernier décret précise ainsi que les décrets français introduits concernant les bibliothèques universitaires ne touchent la BURS que dans la mesure où elle a le caractère d'une bibliothèque universitaire, mais sous réserve des règles qui la régissent en tant que bibliothèque régionale... La situation peu précise de la bibliothèque doit donc être tirée au clair au plus vite.

Dès janvier 1924, le député Charles Frey adresse au recteur un projet de nouveau statut qui s'appuie largement sur les idées déjà développées par Wilhelm dans un mémoire de novembre 1923, à savoir la constitution d'une bibliothèque nationale, au statut analogue sur certains points à celui des bibliothèques Sainte-Geneviève, de l'Arsenal et Mazarine (décret du 7 avril 1887) ${ }^{10}$. Dotée de la personnalité civile, ayant un budget autonome, la bibliothèque serait alors dirigée par un administrateur et un conseil composé de bibliothécaires. Cela assurerait ainsi une indépendance totale par rapport à l'université et éliminerait tout ce qui avait été perçu comme des ingérences (de la part du Conseil de l'université ou de la commission ad hoc existant depuis 1920). La bibliothèque conserverait ainsi son caractère dual, en assurant aux professeurs les facilités et garanties du règlement des bibliothèques uni- versitaires françaises, le contrôle comme avant 1918 de la moitié des acquisitions, tout en maintenant au public non universitaire les avantages concédés jusqu'alors (accès aux salles de lecture, prêt à domicile, etc.).

Le 10 juillet 1924, six députés alsaciens et un député lorrain (Frey, Walter, Altorffer, Scheer, Oberkirch, Seltz, Schuman) se décident à déposer un projet de loi fixant le statut de la bibliothèque universitaire et nationale de Strasbourg, ayant la triple mission d'être le laboratoire de " la pensée française incarnée dans la grande université de Strasbourg ", un foyer de diffusion de la civilisation française dans la population alsacienne et lorraine, et enfin un centre de bibliographie germanique de "l'Europe alliée et amie " ". L'institution doit, pour les députés, conserver son autonomie la plus complète, vue comme une condition essentielle de son développement normal dans l'avenir, tout en conservant des liens « aussi étroits que possibles " avec l'université.

Or parallèlement à ces démarches et suite au décret du 30 mai 1924, le Conseil de l'université s'est emparé de la question dans sa séance du 11 juin 1924 et décide de nommer une commission (composée en grande partie de doyens et de professeurs, dont par exemple Christian Pfister) chargée de donner son avis sur le statut de la bibliothèque et élaborant le règlement universitaire intérieur de l'établissement. Celle-ci se réunit à plusieurs reprises, prenant en compte le projet de loi déposé, et rend son rapport au Conseil en janvier 1925. Après débats (auxquels participe activement Raymond Poincaré, présent en tant que président de la Société des amis de l'Université de Strasbourg et très au courant de la question, compte tenu de ses liens avec Pfister, son ancien camarade de Louis-le-Grand) ${ }^{12}$. Alors que les représentants des facultés de théologie continuent de défendre le principe de l'indépendance, les facultés de droit, sciences, pharmacie et, après hésitations, lettres défendent certes aussi l'idée d'une bibliothèque ayant le caractère d'une bibliothèque nationale (avec l'accès d'un public non universitaire), mais organisée dans le cadre de l'université, sous l'autorité du recteur. L’idée affirmée à plusieurs reprises est qu'une université aussi importante que Strasbourg ne peut vivre sans bibliothèque affectée à son usage. Selon le projet développé, une commission d'achat assisterait l'administrateur et aurait à sa disposition une part importante des crédits d'acquisitions. Le Conseil de l'université délibérerait sur tous les règlements relatifs à la bibliothèque, conformément au décret français du 21 juillet 1897. Il y a donc une opposition absolue aux articles 8 et 9 du projet de loi, prévoyant une administration 


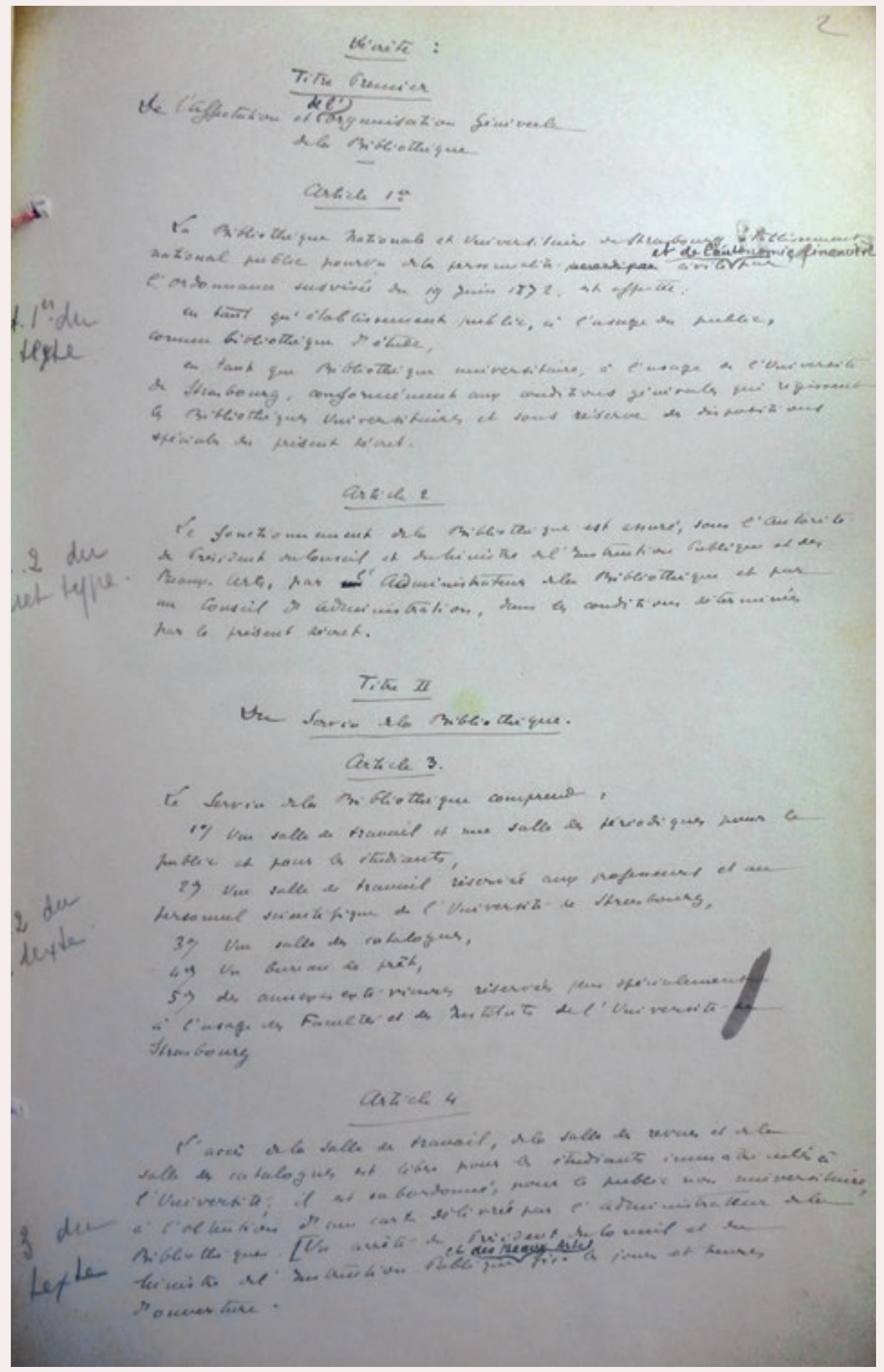

Une version manuscrite du décret avec les ultimes corrections (juillet 1926 ?) (coll. Archives départementales du Bas-Rhin) 


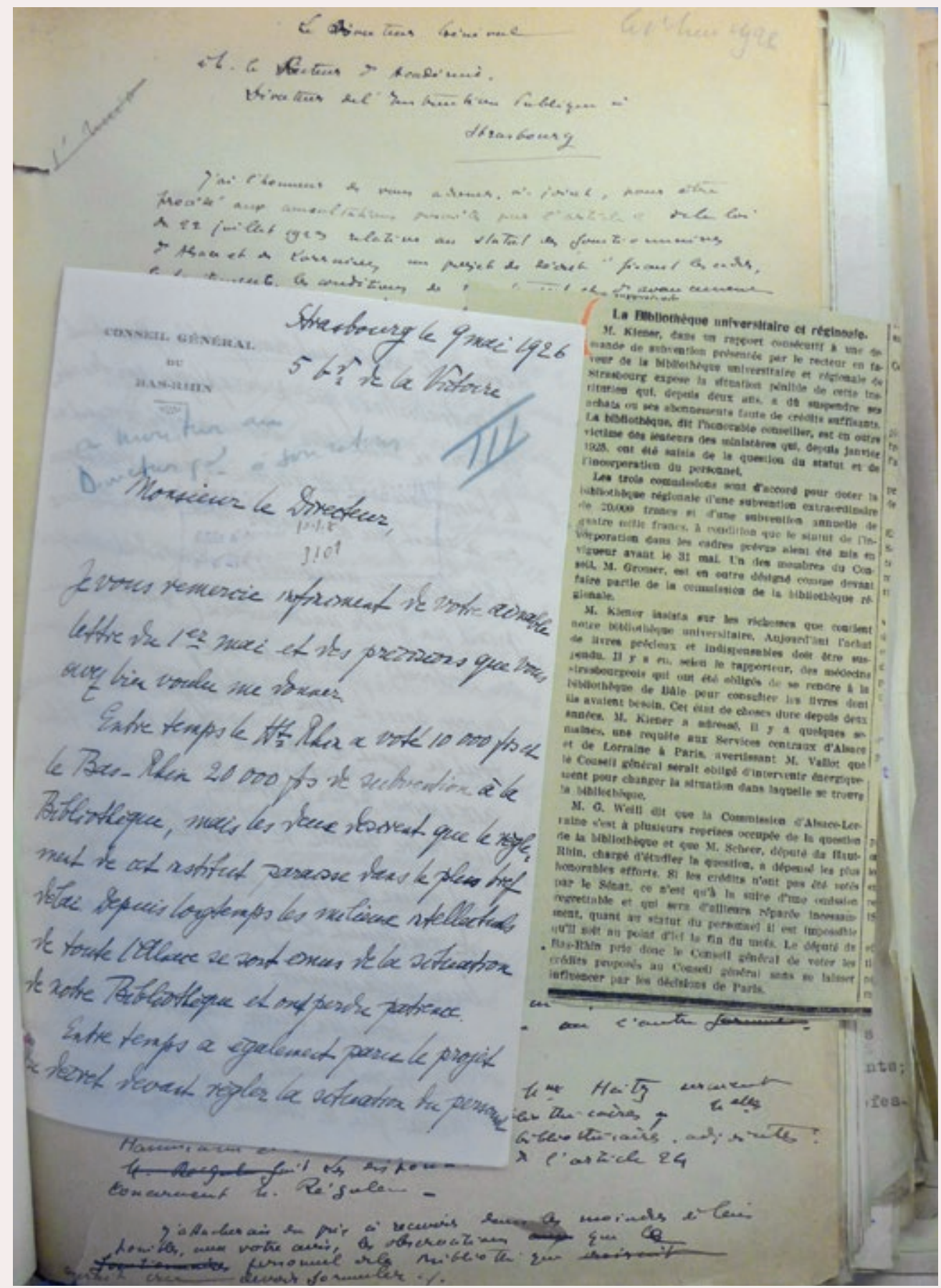

Trois pièces issues du carton ADBR, AL 98,1060, 9 mai 1926, illustrant la polémique sur la bibliothèque (lettre de Fritz Kiener du 9 mai 1926, article de journal rendant compte de la séance du Conseil général le 7 mai, brouillon d'une lettre de la DGSAL au recteur le $1^{\text {er }}$ mai) (coll. Archives départementales du Bas-Rhin) 
confiée aux seuls soins des bibliothécaires, et la volonté de favoriser au contraire un conseil composé en majorité d'universitaires. Selon les idées affirmées, celui-ci contrôlerait ainsi le fonctionnement en garantissant les prérogatives des professeurs (comme celle de l'accès aux magasins, objet d'un fort litige avec la direction de la bibliothèque). Si l'idée d'un afflux de dons et un intérêt renforcé du public alsacien avaient pu plaider pour la solution d'un établissement autonome, la tutelle de l'université apparaîtrait plus susceptible de favoriser un engagement du personnel universitaire envers " sa " bibliothèque. Ce dernier aurait plus la volonté de l'enrichir, au lieu de favoriser le développement de bibliothèques de facultés et d'instituts, développement perçu comme complètement abusif et " insolite " par l'administration ${ }^{13}$. On oppose enfin le financement venant de l'université aux subventions modestes versées par les institutions alsaciennes. Celle-ci devrait donc devenir le tuteur et le " banquier " de la bibliothèque, et éviter à celle-ci un déclin et une rivalité inévitable et inutile avec l'université ${ }^{14}$.

Malgré cette opposition, on constate cependant un accord unanime en faveur d'un établissement public national bien différent d'une bibliothèque universitaire française classique, compte tenu des origines de la bibliothèque, de son fonctionnement (en particulier son système de prêt jugé unique en France) et de la richesse et de la diversité de ses collections, constituant un patrimoine national. Un accord existe également sur le caractère dual de l'établissement, à la fois universitaire et ouvert au public érudit local (même si un débat existe au sein de l'université sur la restriction de l'accès à la salle de travail au public non universitaire, la gratuité accordée à celui-ci et la restriction des facilités du prêt, au grand dam de l'autre camp dont certains à l'inverse veulent limiter les facilités et les commodités dont bénéficient les universitaires).

Il revient donc à l'administration de trouver un compromis dans un contexte très défavorable. L'arrivée au pouvoir du Bloc des gauches et sa politique alsacienne attisent les tensions et avivent les susceptibilités. L'instabilité politique et financière accapare les attentions alors même qu'en 1925, la fin du Commissariat général d'AlsaceLorraine et la mise en place d'une Direction générale des services d'Alsace et de Lorraine (DGSAL ${ }^{15}$ ) suscitent des retards et la nécessité pour les administrations de fixer leurs relations réciproques (notamment entre le ministère de l'Instruction publique, la DGSAL et le recteur). Les travaux de l'administration sont ralentis du fait du dépôt du projet de loi et de la nécessité d'attendre que les commissions parlementaires d'Alsace-Lorraine débordées et les rapporteurs s'en saisissent. De plus, la préparation et la complexité du décret fixant les cadres de la bibliothèque et l'incorporation de ceux-ci dans les cadres généraux perturbent le règlement de la question ${ }^{16}$. L'inquiétude du personnel aboutit à un grand nombre de démarches directes et indirectes via le personnel politique, qui retardent l'avancement de la question, les dossiers étant liés. Le retard pris par les décrets aboutit à une précarisation croissante du personnel face à l'inflation et à l'impossibilité de profiter des augmentations décidées pour l'ensemble des fonctionnaires ${ }^{17}$. Cette précarisation gagne également, comme on l'a évoqué, la bibliothèque dans ses moyens de fonctionnement, avec la chute de plus en plus importante des acquisitions. L'opinion a tendance alors à lier (à tort) cette situation avec l'absence de statut et l'impossibilité de disposer de ressources financières autres que les subsides de l'État ${ }^{18}$.

\section{Une solution de compromis}

En tant que représentante du ministère de l'Instruction publique, l'Inspection générale des bibliothèques, par l'intermédiaire de son représentant Vidier, va jouer un rôle fondamental mais ingrat dans la recherche d'un compromis nécessaire et évitant les mesures extrémistes. Elle seconde ainsi utilement l'action du recteur Charlety et de Paul Valot, le directeur emblématique de la DGSAL pendant presque tout l'entre-deux-guerres, qui a su obtenir la confiance des milieux politiques alsaciens tout en essayant de coordonner au mieux les relations entre administrations. Pour les raisons évoquées plus haut, il faut attendre janvier 1926 pour que la DGSAL, le rectorat et l'IGB (Vidier) représentant l'Instruction publique accélèrent leur travail de coordination sur les textes et cherchent à sortir de la rivalité opposant l'université et les députés alsaciens.

Fin avril 1926, Paul Valot arrive à un compromis avec les députés alsaciens sur un projet qui, selon lui, rétablit dans ses grandes lignes le régime antérieur à l'armistice. Utilisant les possibilités ouvertes par la loi du 17 octobre 1919 (possibilité de légiférer par décret pour l'adaptation des lois et règlements locaux alsaciens et l'introduction de dispositions législatives françaises), il parvient à favoriser la voie réglementaire, l'adoption d'un décret semblant plus rapide qu'un projet de loi dont la seule nécessité repose sur l'octroi de la personnalité civile de 
la bibliothèque. Le ministère de l'Instruction publique se montre alors réticent à favoriser le vote d'une loi pour Strasbourg alors que le projet concernant les bibliothèques nationales parisiennes (Bibliothèque nationale, Arsenal, Sainte-Geneviève, Mazarine) n'est pas encore voté $^{19}$. La prise en compte du fait que l'ordonnance de 1872 n'a jamais été abolie favorise heureusement cette solution, le texte visant non à créer un établissement nouveau mais à le réorganiser et à l'adapter au contexte français. Sur le plan juridique, la solution réglementaire établit ainsi la continuité avec l'époque précédente et ne crée pas une nouvelle institution. Les députés alsaciens s'engagent alors à retirer leur projet de loi à condition que le texte du futur décret soit jugé compatible avec les grands principes à la base du projet de $10 i^{20}$.

Tout en continuant de reconnaître le caractère national de l'établissement, les députés alsaciens obtiennent également comme concession qu'il continue à s'appeler "bibliothèque universitaire et régionale ". Mais suite à l'opposition déterminée de Vidier ${ }^{21}$, Valot finit par céder in extremis en juin : la bibliothèque sera bien " nationale et universitaire $"{ }^{22}$.

Au-delà du débat sur la dénomination à adopter, le caractère dual de la bibliothèque a été vite tranché. En tant que bibliothèque universitaire (appliquant les règles françaises générales concernant ce type de bibliothèques, en dehors des dispositions spéciales du statut) et en tant que bibliothèque d'étude pour le public, elle accueille les professeurs et les étudiants inscrits (avec reversement par l'université d'une partie des droits de bibliothèque), ainsi que le grand public, en établissant pour ce dernier des cartes (afin de pouvoir réguler l'accès en cas de problème particulier). Le prêt reste ouvert, le ministère acceptant cependant de maintenir provisoirement les règles en vigueur, c'est-à-dire celles rendant depuis 1919 le prêt payant pour les étudiants et les particuliers, afin de ne pas priver l'établissement d'une recette importante ${ }^{23}$.

Le débat principal concerne finalement la composition du conseil d'administration (cette appellation prévaut sur celle de comité consultatif évoquée dans certains projets), composé non pas exclusivement de bibliothécaires (ce qui affaiblirait aussi, pour l'IGB, l'autorité de l'administrateur), mais sans majorité pour le monde universitaire (en évitant ainsi de faire de l'administrateur un simple " agent d'exécution » des représentants de l'université). Cette dernière sera donc représentée par le recteur et les six doyens. Les députés alsaciens obtiennent alors que le nombre de personnes "notables " (ne pouvant appartenir ni à l'université ni au monde des bibliothèques), désignées par le ministère, soit porté de quatre à sept (puis rapidement à neuf en 1927, puis dix en 1933), afin d'obtenir la parité. Ce seront, en très grande majorité, des députés et sénateurs d'Alsace-Lorraine (dont ceux ayant le plus participé à l'élaboration du statut, comme le pasteur et député Scheer, rapporteur du projet de loi, qui sera l'interlocuteur officieux de l'administration). L'administrateur, un représentant du personnel, l'IGB et le directeur de la DGSAL complètent les membres du conseil d'administration (en 1927 s'ajoutant un représentant du Chapitre de Saint-Thomas) ${ }^{24}$.

En fonction du même principe, les députés alsaciens s'opposent également à ce que le recteur préside le conseil d'administration. Le président et le vice-président seront finalement choisis obligatoirement parmi les personnalités extérieures. Après discussions, le ministère et la DGSAL pressentent Raymond Poincaré comme futur président. Poincaré acceptera la proposition, même si ses occupations politiques l'empêcheront presque toujours d'être présent à Strasbourg, la présidence de facto étant assuré le plus souvent par Alfred Coville, vice-président et fonctionnaire de l'Instruction publique (Direction de l'enseignement supérieur). De manière plus curieuse, en se basant sur la situation existant avant 1918, à défaut d'un bibliothécaire comme secrétaire trésorier (comme prévu dans le projet de loi), les députés obtiennent également que la bibliothèque ait non pas un comptable spécial à l'établissement mais un comptable commun avec l'université, comme c'était le cas auparavant.

Il n'en reste pas moins que, à la différence du système allemand (budget inclus dans celui de l'État d'Alsace-Lorraine, droit de disposition du patrimoine mobilier et immobilier par l'État), c'est le nouveau conseil d'administration qui délibère sur le budget (arrêté finalement par l'État) et accepte les dons et legs. Il arrête également une liste des acquisitions représentant la moitié des crédits (l'autre moitié relevant directement de l'administrateur).

Le décret sur le statut subit finalement un dernier retard face aux réactions de dépit que suscite chez certains le projet parallèle de décret concernant le personnel. Ce dernier ne perd pas la qualité de fonctionnaire d'État, malgré l'autonomie de l'établissement. En dépit des tentatives de certaines administrations, l'IGB a obtenu le maintien de douze postes de bibliothécaires et de huit postes de gardiens (au lieu de 4, le nombre d'agents administratifs passant de 7 à 1), l'effectif total étant de 22 (au lieu de 24 en 1918). Le texte cherche à régler un certain nombre de situations particulières par 
assimilation. Mais les avantages concédés à certains (par exemple un reclassement sur mesure comme bibliothécaire du responsable de l'ancienne bibliothèque administrative) suscitent les critiques des autres (ainsi les " bibliothécaires adjointes " reclassées dans ce grade spécial mais invoquant leur diplômes pour devenir aussi bibliothécaires) et aboutissent à de nouvelles démarches auprès de l'administration. La question des traitements est également débattue : les bibliothécaires de droit local (recrutés à l'époque allemande parmi les professeurs d'enseignement secondaire) revendiquent des échelles de traitement analogues à celles de ces derniers et non à celles, inférieures, des bibliothécaires français.

Après de derniers arbitrages et un dernier retard lié à la crise ministérielle qui est en train de mettre fin au "Cartel des gauches ", le texte final des deux décrets est validé par le ministère des Finances et fait l'objet pour les détails d'une rédaction définitive.

Les signatures, le 23 juillet du décret " relatif à l'administration et au fonctionnement de la bibliothèque nationale et universitaire de Strasbourg ", et le 28 juillet du décret " fixant les cadres, traitements, conditions de recrutement et d'avancement et le régime disciplinaire et portant reclassement et incorporation du personnel de la bibliothèque nationale et universitaire " donnent enfin à l'établissement la stabilité tant recherchée. Elles le font véritablement entrer dans un cadre français qui va lui permettre en partie de reprendre son développement. Il s'agit cependant d'un compromis illustrant la position toujours un peu instable de la bibliothèque par rapport à l'université : malgré le cadre offert par le statut, la liaison toujours difficile entre la nouvelle BNU et les bibliothèques d'instituts en sera un bon exemple. Le débat sur le statut offre finalement une très bonne illustration des attentes et des craintes des différents acteurs du site strasbourgeois, et permet de mieux comprendre les origines des débats que connaîtra l'évolution de la bibliothèque dans la seconde moitié du $20^{\mathrm{e}}$ siècle.

\section{Julien Gueslin}

\section{Notes}

1 - Laurence Buchholzer, " De l'Allemagne à la France (1918-1939) ", in Frédéric Barbier (dir.), bibliothèques strasbourg, origines-XXI siècle, Strasbourg : BNU ; Paris, Édition des Cendres, 2015, p. 259-290

2 - Dans les archives de la BNU (= ABNU) se trouve un carton AL 101,232-236, issu des archives du rectorat et qui a donc fait l'objet d'un classement au sein des Archives départementales, avant de revenir à une date inconnue dans les archives de l'établissement.

Plus important, au sein du fonds dit Valot (Direction générale des services d'Alsace et de Lorraine) se trouvent un certain nombre de cartons jusque-là non encore inventoriés, dont les cartons AL 98,1060 à 1063 concernant la tutelle et la gestion directe de la bibliothèque entre 1925 et 1938 par les services centraux de l'État. Dans le carton 106o se trouve le dossier traitant de l'élaboration des textes législatifs et plus particulièrement de ceux des décrets de 1926.

3 - AL 98,1062 (lettre du 12 avril 1926)

4 - Lettres de Klein (24 juin 1919) et notice de Wilhelm (19 novembre 1923), $\mathrm{ABNU}, \mathrm{AL}$ 101,232 (rapports) et 236 (règlement).

5- ADBR, AL 98,106o, dossier Textes législatifs (30 avril 1926)

6 - KULB : Kaiserliche Universitäts- und Landesbibliothek zu Strassburg, nom de la BNU jusqu'en 1918.

7 - Buchholzer, op. cit., p. 267 et AL 121,1070 (lettre de Millerand du 10 mai 1919)

8 - Il se place donc dans la hiérarchie entre le directeur de la KULB et le secrétaire d'État pour l'Alsace-Lorraine. Ce dispositif existait depuis 1909, la découverte de problèmes comptables ayant incité l'État à renforcer le système de contrôle (auparavant un simple fonctionnaire du ministère assurait le contrôle et jouait le rôle d'intermédiaire).

9 - Voir note 7

10 - AL 101,236 / Dossier Règlement (sous-dossier Projet de statut / Rapport), lettre du 24 janvier 1924

11 - Proposition de loi $\mathrm{n}^{\circ}$ 266, annexée au procès-verbal de la deuxième séance du 10 juillet 1924

12 - Voir Antoine Gaugler, " Les Amis des Étudiants de l'Université de Strasbourg (1929) ", in Bulletin de la Société des Amis des Universités de l'Académie de Strasbourg, $\mathrm{n}^{\circ}$ 70, 2016 (à paraître)

13 - ADBR, AL 98,106o, Dossier Textes législatifs (rapport confidentiel de Vidier du 10 mars 1925, remis le 27 janvier 1926)

14 - AL 101,236 / Dossier Règlement (sous-dossier Bibliothèque universitaire. Projet de statut, lettres des 5 et 10 juillet 1924 et passim)

15 - Celle-ci est partagée entre services centraux à Paris et services alsaciens (dont ceux de l'Instruction publique)

16 - Un premier projet d'incorporation est présenté par le rectorat au personnel en octobre 1924, puis envoyé à l'Instruction publique en février 1925, mais ne fait l'objet d'un échange avec la DGSAL qu'en janvier 1926. Une nouvelle version envoyée en mai 1926 suscite alors de vives protestations avant d'ultimes arbitrages (AL 98,106o, dossier Textes législatifs, passim)

17 - ADBR, AL 98,106o (25 mars 1926) et 1062 (3 et 18 mars, 12 avril, 8 juin 1926)

18 - Voir l'échange entre F. Kiener et Paul Valot : AL 98,1060, dossier Textes législatifs (20 avril et $1^{\text {er }}$ mai 1926), et les débats du Conseil général du BasRhin dans sa séance du 7 mai (ADBR, AL 98,1060).

19 - En attendant le vote d'une loi commune, des mesures transitoires auraient permis à la BURS de recevoir subventions, dons, etc., et de faire certaines recettes sous le couvert de l'université.

20 - ADBR, AL 98,106o, dossier Textes législatifs (11 janvier, 3 février 1926)

21 - Ibid., 8 et 15 juin 1926 : Vidier fait remarquer que la bibliothèque de Strasbourg a toujours été une bibliothèque d'État (Reichsland puis France), invoque hors de Paris le cas de la Bibliothèque nationale se trouvant déjà à Alger, et enfin l'absurdité sinon l'illégalité à qualifier un établissement national de " régional ».

22 - Ibid., 30 avril, 2, 14 et 15 juin 1926

23 - Sous le régime allemand, le prêt était payant (redevance) pour tout le monde. En 1919, un règlement en avait exonéré les universitaires mais pas le reste du public. Voir la note suivante.

24- ADBR, AL 98,106o, dossier Textes législatifs (rapport confidentiel de Vidier du 10 mars 1925 remis le 27 janvier 1926, note du 30 avril 1926) 